\title{
Article \\ Instability of Traveling Pulses in Nonlinear Diffusion-Type Problems and Method to Obtain Bottom-Part Spectrum of Schrödinger Equation with Complicated Potential
}

\author{
Michael I. Tribelsky ${ }^{1,2}$ (D)
}

1 M. V. Lomonosov Moscow State University, Faculty of Physics, Moscow, 119991, Russia; mitribel@gmail.com

2 National Research Nuclear University MEPhI (Moscow Engineering Physics Institute), Moscow, 115409, Russia

\begin{abstract}
The instability of traveling pulses in nonlinear diffusion problems is inspected on the example of Gunn domains in semiconductors. Mathematically the problem is reduced to the calculation of the "energy" of the ground state in Schrödinger equation with a complicated potential. A general method to obtain the bottom-part spectrum of such equations based on the approximation of the potential by square wells is proposed and applied. Possible generalization of the approach to other types of nonlinear diffusion equations is discussed.
\end{abstract}

Keywords: nonlinear diffusion; traveling waves; stability; Goldstone modes; Schrödinger equation; spectrum of low-exited states.

\section{Historical Remarks}

When the Editors kindly offered me to submit a paper to this Special Issue dedicated to my fifty years in physics, I began to think about a possible topic of the paper. Finally, I decided that the best is to generalize the results of my very first paper [1], which formally was published exactly fifty years ago. I said "formally" because actually this paper has never been published. Perhaps, its story is so remarkable that it is worth telling it here.

The point is that though I graduated from the Lomonosov Moscow State University (MSU) - the one where now I head a laboratory - I did not enter this university in the usual, standard manner. It so happened that the university I entered was the Belorussian State University (BSU) in Minsk. Now Minsk is the capital of independent state Belarus, while at that time, Minsk and Moscow both belonged to a single state: the Soviet Union. In Minsk, I met my first scientific adviser Prof. Mikhail Aleksandrovich El'yashevich [2].

Then, upon completing my first two university years in Minsk, I moved to Moscow. Thus, I became a student of MSU due to my transfer from BSU. Just one letter difference in the names meant the drastic difference in the ranks. Though BSU was quite a good university, MSU was (and is) the Number One.

Doing paperwork related to the transfer, I asked Prof. El'yashevich for a reference letter to somebody of his collaborators in Moscow. I got this letter to his former Ph.D. student Dr. Sergei Ivanovich Anisimov [3], who became my next scientific adviser.

It was 1969. At that time, I could not even imagine how lucky I was. Dr. Anisimov was employed by the Landau Institute for Theoretical Physics. The Institute was created just five years ago to collect "under a single roof" the first generation of Prof. Landau's disciples [4]. By the time I am talking about, all of them have become first magnitude stars in the scientific sky.

Thus, suddenly and almost by chance, I occurred embedded in the scientific atmosphere representing the very top of theoretical physics in the USSR, and I would say in the entire world too. Moreover, I got another piece of good luck, though, I did not know about it yet: In the very same year of my transfer to MSU, a prominent theoretical 
physicist Prof. Il'ya Mikhailovich Lifshits [5] succeeded late Prof. Landau's position of the Head of the Theoretical Physics Department at the Kapitza Institute [6]. To this end, he moved to Moscow from Khar'kov (a big Ukrainian city), where he resided before. In addition to this position, Il'ya Mikhailovich got a professorship at the Chair for Quantum Theory, the Faculty of Physics, MSU. By that time, another employee of the Landau Institute and a disciple of Il'ya Mikhailovich, namely Dr. Mark Yakovlevich Azbel [7] already shared his position at the Landau Institute with a professorial position at this Chair. The second disciple of Il'ya Mikhailovich, who came from Khar'kov to Moscow and became a Professor of the same Chair, was Dr. Moisei Isaakovich Kaganov. Among other scientific accomplishments of this group was the galvanomagnetic theory of electrons with an arbitrary dispersion law. The theory describes the effects of both electric and magnetic fields acting together on free electrons in metals. In this theory, electrons are regarded as quasi-classical particles, but instead of the conventional dependence of their energy on (quasi)momentum $\varepsilon(p)=p^{2} /(2 m)$ this dependence may be arbitrary. The theory was a breakthrough in the quantum theory of solids, and was named after its creators the LAK-theory (Lifshitz, Azbel, Kaganov) ${ }^{1}$. On top of that, I should add that the Chair for Quantum Theory was headed by one of the most prominent experts in theoretical physics, a very respectable person with the highest moral standards, Academician of the Soviet Academy of Sciences, Prof. Mikhail Aleksandrovich Leontovich [8]. Alas, all of them have passed away already...

In 1969 I did not know anything of these persons and the Chair, but Dr. Anisimov did. Therefore, when I asked his advice about the specific Chair at the Faculty of Physics for my specialization, he immediately replied, "The one where I.M. Lifshitz is a Professor." So I did, and in addition to the excellent external scientific environment in the Landau Institute, I got benefits from the one at the Chair for Quantum Theory.

Soon after my appearance at the Chair, I began to attend lectures on the quantum theory of metals given by Prof. Kaganov. Bearing in mind that Prof. Kaganov was a brilliant lecturer, it is easy to understand that I admired the beauty of the lectures and that of the theory as a whole. Thus, it is easy to understand that when Anisimov asked me about the preferences for the topic of the future study, my reply was, "Something from the quantum solid-state physics."

It is worth mentioning that at the time, I did not have any idea about the specific subfield, where the accomplishments of Dr. Anisimov lay (laser-matter interaction, physical hydrodynamics, shock waves, plasma physics and alike). Fortunately, he was a physicist with broad interests and understood physics far beyond the frames of his own subfield. It was a typical feature of physicists from the Landau Institute originated in Prof. Landau himself: Broad knowledge helps to see cross-links between different, seemingly not connected to each other, problems. This, in turn, sometimes helps to obtain very beautiful and unexpected results.

Thus, according to my desire, Dr. Anisimov posed me a problem from the quantum solid-state physics. It was related to the appealing at that time Gunn effect in semiconductors [9]. At that time, the effect was a fascinating, challenging topic, and up to now, it still attracts a great deal of attention from researchers [10-15].

Naturally, now the understanding of the effect is more profound, and its mathematical description is much more elaborated than it was 50 years ago, see, e.g., Ref. [16]. However, since the goal of this paper is to generalize the methods and results discussed in Ref. [1], making them applicable to a broad class of related problems, rather than to inspect specific peculiarities of the Gunn effect itself, in what follows I stick to the old model of the effect [17] employed by Knight and Peterson [18] and then, in my paper [1].

Very briefly, the essence of the phenomenon is as follows. In a strong enough electric field $\mathbf{E}$ applied to a semiconductor, the conductivity of the sample depends on $\mathbf{E}$ and

1 There is an interesting story related to this abbreviation. When another Prof. Landau's disciple, Prof. Alexander Solomonovich Kompaneetz, known, in addition to his outstanding scientific results, for his sense of humor, leant about LAK-theory, he said, "It is excellent that the authors did not employ the inverted order of them." (kal in Russian means excrements.) 
the current-voltage curve becomes nonlinear. In some cases, calculations based on the assumption of the spatially homogeneous distribution of $\mathbf{j}$ and $\mathbf{E}$ along and across the sample gives rise to very unusual behavior of this dependence so that in a certain area of the $\mathbf{E}$ values an increase in $\mathbf{E}$ results in a decrease in $\mathbf{j}$, where $\mathbf{j}$ stands for the current density.

In what follows only one-dimensional cases will be considered, so I can replace $\mathbf{j}(\mathbf{E}) \rightarrow j(E)$. Then, by definition, the conductivity $\sigma=j / E$. Let us define the differential conductivity as $\sigma_{d}=d j / d E$. Thus, the area mentioned above is characterized with a negative differential conductivity. Here I will not discuss the microscopic mechanisms explaining the negativeness of $\sigma_{d}$; their detailed description may be found, e.g., in Ref. [19]. Further increase in $E$ makes $\sigma_{d}$ positive again so that the overall shape of the current-voltage curve resembles the letter " $N$ ".

It occurs that the assumption about the spatially uniform distribution of $j$ and $E$ in the regions with $\sigma_{d}<0$ is erroneous. This distribution is unstable against small spatially-inhomogeneous perturbations and, eventually, is destroyed owing to their growth. In certain cases, the instability ends up forming a strong field domain bounded by the corresponding layers of charge density. The domain drifts along the sample with a constant speed until it hits the sample edge (anode). There the domain disintegrates, a new one emerges at the opposite side of the sample, and the process repeats. As a result, oscillations with the period $L / v$ are generated. Here $L$ stands for the sample length and $v$ is the drift speed of the domain. This is the Gunn effect [9]. It is successfully used in Gunn diodes to generate microwave oscillations [19].

Let us consider an idealized case of a single traveling strong-field Gunn domain drifting with a constant velocity along an infinitely-large sample. The "strong-field domain" means that the field outside it equals $E_{1}$ (see Fig. 2 below), while inside the domain it is greater than that. If the voltage applied to the sample is constant, the single domain with a fixed shape is stable, while a configuration with several domains is not. However, if an external source fixes the current in the sample, even the single domain becomes unstable. The instability affects the faces of the domain, which begin to move in opposite directions with respect to the center of the traveling domain. If they move to each other, the domain contracts and, eventually, collapses. If the faces move in the opposite direction, the domain expands and transforms into two traveling layers [19].

Linear analysis of this secondary instability of a single traveling domain at a fixed current in the circuit was performed by Knight and Peterson [18]. Mathematically the stability problem was reduced to the calculation of a gap between the ground and the first excited states in the 1D Schrödinger equation with a complicated potential (see below). To this end, Knight and Peterson employed the WKB-approximation. However, this approximation is accurate for highly excited states when the characteristic spatial scale of the wave function oscillations is small relative to the one for the variations of the potential. This is not the case for the ground state. Hence, the accuracy of the results obtained in Ref. [18] through the WKB-methods, at least, was questionable. The problem posed for me by Dr. Anisimiv was to check the results of Knight and Peterson employing for the calculations an approximation different from WKB.

If I faced this problem now, quite probably, I would have used the Ritz method supplemented by the orthogonality condition of the wave functions of the ground and excited states [20]. However, at that time, I was much more ignorant than I am now. Therefore, instead of taking a simple, well-known way (perhaps, at that time, it was neither simple nor well-known for me), I decided to go on my own one. Specifically, I decided no less than to find a new method to obtain approximate solutions to the Schrödinger equation opposite to the WKB-method, which could be suitable for the ground and low-excited states. Furthermore, I succeeded to do that! So, maybe ignorance is not always bad.

The main idea of the developed approach is somewhat unusual for quantum mechanics, where approximations conventionally are targeted to a wave function, while 
the potential is given and fixed. But, on the other hand, if we have ground and lowexcited states in a complicated potential, the potential has a sharply varying profile relative to that of the wave functions, and the latter is not very sensitive to the fine details of the former. If so, why one does not try to approximate the potential, with some simple shapes, say, with square wells? Then, the Schrödinger equation becomes either exactly solvable or readily treated by perturbation methods.

The most challenging task was to set the first step in this way. The rest was just a matter of not so complicated calculations. Shortly I did them and presented the results to Anisimov. "Very well," he said, "the problem is solved. Write a paper. One more point to be made. Il'ya Mikhailovich Lifshitz, has organized a periodic scientific seminar at your Faculty. It takes place every second and fourth Thursday of a month from September to June. I recommend you to contact Il'ya Mikhailovich and ask him to put your talk about this study in the seminar program."

Up to now, I remember how difficult it was for me (a fourth-year undergraduate student) to approach such famous scientist as Il'ya Mikhailovich was and request a talk at his seminar. Finally, I collected all my courage and did it. "Excellent," replied Il'ya Mikhailovich, "Please contact the seminar's secretary, Mr. Rzhevsky and ask him to find the nearest free spot in the program. Will 45 minutes be enough for you?"

To give a 45-minute talk in front of an audience of top-rank experts, including a dozen of world-class scientists! My knees turned to jelly, but there was no way to retreat.

It is remarkable that I vividly remember any moment before and after my talk, but nothing of the talk itself. However, it seems that I stood this test. Moreover, the talk at this seminar was a milestone for my relationship with Il'ya Mikhailovich. Since then, every my new result was discussed with him, either through a talk at the seminar or in a private manner at his office at the Kapitza Institute. Later on, I became a close associate and coauthor of Il'ya Mikhailovich [21]. We even had a joint Ph.D. student. Our close contacted lasted until his unexpected premature death of a heart attack in 1982.

However, all this will be later on. At the time I am talking, I could not imagine even a small part of that.

Thus, the first task (the talk) was complete, but the second remained: I had to write a paper. It was my very first paper, and it took a lot of my time and effort to do that. Finally, rather an extended manuscript (in Rusian) was submitted to Fizika i Tekhnika Poluprovodnikov (Physics and Technology of Semiconductors).

It was the beginning of bad luck for this paper. At that time, a new publication option was introduced. For some papers (especially lengthy ones), only abstracts were published. The papers themselves were deposited in specially assigned institutions. If an abstract of such a paper drew somebody's attention and he/she was interested in the complete text, a copy of the one could be posted to him/her upon a request. Maybe it was an attempt to reduce the printed size of scientific journals and to solve, at least partly, the eternal Soviet problem of the paper deficit. Anyway, my paper was accepted under this condition. Its abstract was published, see Fig. 1, while the full text was deposited at RED Electronics.

Since that, many events have happened. The country named the Soviet Union does not exist anymore. Regarding RED Electronics, I am afraid it has shared the destiny of the country. Now I am residing within walking distance from the building where $R \mathcal{E} D$ Electronics was situated. It is a shopping mall. Therefore, it is quite probable that the full 


\title{
ОБ ИНКРЕМЕНТЕ НЕУСТОЙЧИВОСТИ ГАННОВСКІХ ДОМЕНОВ В РЕЖИМЕ ПОСТОЯННОГО ТОКА
}

\section{М. И. Трибельский}

Исследуется развитие неустойчивости в широких ганновских доменах (ширина верпины много больше ппирины фронтов) в режиме стационарного внешнего тока. В основу расчетов положена феноменологическая модель, в которой полный ток складывается из тока проводимости и диффузионного тока. Неустойчивость заключаетея в том, что фронты домена начинают смещатьея в противоположных паправлениях. Вычислен инкремент нарастания неустойчиности. Коэффициент диффузии предполагался независящим от поля. Задача математически свелась $\mathrm{k}$ решению уравнения типа одномерного уравнения Шредингера с некоторым сложным потенциалом. Показано, что результаты слабо зависят от вида этого потенциала. Поэтому он был заменен потенциалом в виде двух прямоугольных ям, что позволило выполиить расчеты в конечном виде. Задача решена в двух предельных случаях: симметричного и сильно несимметричного доменов. В обоих случаях вычислены дополнительное падение напряжения на домене, а также ширина фронтов и вершины домена.

Сушественно, тто в конечный результат не входят какие-либо интегральные характеристики. Он зависит только от значения функций $j(E)$ и $d j / d E \equiv \sigma_{d}$ в некоторых характерных точках. Развитый метод без существенных изменений может быть применен к исследованию характера неустойчивости волн в виде двух и более доменов.

Поступило в Редакцию 13 марта 1971 г.

Figure 1. The only ever published piece of paper [1].

text of my paper has ended up in a nearby scrap-heap, and the abstract reproduced in Fig. 1 is the only remained piece of the paper ${ }^{2}$.

The next attempt to publish these results I made after the defense of my MS thesis. An appointed referee of the thesis was another disciple of Prof. Landau, Prof. Igor Ekhiel'evich Dzyaloshinskii [22] ${ }^{3}$. After reading the thesis, he said that the results of this level should be available to the international community, and I should publish them abroad in English.

Well,... it was easier to say than to do. To say nothing about poor English, which I had at that time, sending a scientific paper abroad from the Soviet Union was not simple at all. The authors themselves were not eligible to do that. A manuscript had to be sent through specially authorized personnel. The personnel decided whether or not the

\footnotetext{
2 Here is the translation of the Russian text shown inFig. 1
}

Vol. 10 DE-416 dated June 30, 1971

On the increment of the instability of the Gunn domains in the DC regime

$$
\text { M. I. Tribelsky }
$$

\begin{abstract}
The growth of instability of wide Gunn domains (the width of the top is much larger than the widths of the faces) at the stationary external current regime is inspected. The basis of calculations is the phenomenological model, in which the total current is composed of the conductivity current and the diffusion one. Instability affects the domain faces so that they begin to shift in opposite directions. The instability increment is calculated. The diffusion coefficient is supposed to be independent of the field. Mathematically the stability problem is reduced to a one-dimensional Schrödinger equation with a certain complicated potential. It is shown that the results are weakly dependent on details of this potential. Therefore, the potential is approximated by two square potential wells (separated by a barrier), which made it possible to obtain an explicit expression for the increment. The problem is solved in two limiting cases, namely symmetric and highly asymmetric domains. In both cases, additional drops of voltage on the domain are calculated, as well as the width of the faces and the top of the domain. It is essential that the final result does not include any integral characteristics of the problem. It depends only on the value of the functions $j(E)$ and $d j / d E=\sigma_{d}$ at certain characteristic points. A developed method without significant changes may be extended to the study of the instability of waves in the form of two or more domains.
\end{abstract}


paper could be submitted abroad and, if the decision was affirmative, took care of the submission.

Moreover, prior to the acceptance of the manuscript by the personnel, the authors had to do plenty of paperwork. On top of that, it took two - three months on average for mail to be delivered to the addressee. Up to now, I wonder why it was so. Even if horses delivered the mail, it would not have taken so long time!

I discussed the matter with Dr. Anisimov, and we decided to submit the paper to East-German journal Physica Status Solidi published in English. There were two reasons for this choice. First, sending a paper to an Eastern Block country required less paperwork, and chances to get permission for the submission were higher than that in the case of a Western journal. Second, the requirement for the English quality in this journal was not so strict as those in the West. The latter was important since my English was far from being perfect.

Thus, I wrote in English an elaborated version of Ref. [1] including some new results, did all required paperwork, gave the bunch of documents to the "authorized personnel," and... lost control over the submission. Half a year elapsed, but I had not heard anything from the Editors. Then, I sent to Physica Status Solidi a postcard asking for the status of my paper. A reply came surprisingly fast - just in four months. However, it was pretty unexpected. The Editors informed me that they had never received my manuscript.

By that time, on the one hand, I had already published paper [23], where the secondary instability of the Gunn domain was inspected just employing the Ritz method. On the other hand, I got a job and, owing to that, was forced to abandon my study in solid-state physics and focus on an entirely different topic.

Eventually, the results discussed in Ref. [1] have remained unpublished. Now, fifty years later, I try to realize the advice of Prof. Dzyaloshinskii and make these results available to the international community. Perhaps fifty years is a too long period to complete a task, but "that is not lost that comes at last!"

In the end of this, maybe longsome remarks, I have to say that the results discussed below are not exactly the same as those in Ref. [1]. Firstly, it is not good to publish the same results twice, even if fifty years lie between the two publications. Secondly, I could not do this, even if I wanted to - the original manuscript is lost, and I do not remember all details. Last but not least: now I am a bit more experienced and educated than I was fifty years ago. Therefore, I extracted from this old problem the essential points and generalized them. These points are as follows: (i) the conclusion about the instability of traveling pulses in a broad class of nonlinear diffusion-type problems and (ii) a new method to obtain the bottom-part spectrum of the Schrödinger equation with complicated potential. A discussion of these two issues is presented below.

\section{Problem Formulation}

Thus, the problem is to find the instability increment for a single traveling Gunn domain at a fixed current in the circuit. According to what has been said above, the current-voltage characteristic of the semiconductor sample in question has the shape schematically shown in Fig. 2 . Regarding the external current $j_{\text {ext }}$, I suppose that it satisfies the restrictions $j_{c 1}<j_{\text {ext }}<j_{c 2}$ so that the equation $j(E)=j_{\text {ext }}$ always has tree roots $E_{1,2,3}$.

It is important to stress that the curve presented in Fig. 2 is not the actual currentvoltage characteristic of the sample. As mentioned above, it would have been the one provided $E=$ const along and across the sample. Obviously, this is not the case for the traveling domain, when $E$ is coordinate- and time-dependent. Therefore, only the stable branches of the presented curve with $\sigma_{d}>0$ coincide with the actual current-voltage characteristic. In contrast, the whole curve in Fig. 2 should be regarded as the field dependence of the normalized average electron drift velocity [16]. 


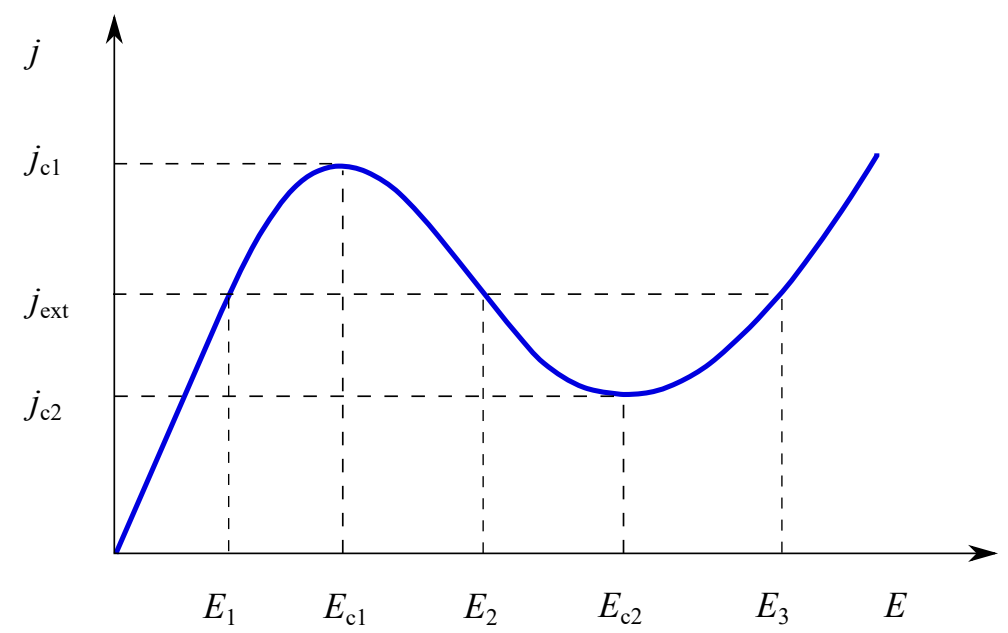

Figure 2. The $N$ type current-voltage characteristics obtained under the assumption that $E=$ const along and across the sample: $\sigma_{d}<0$ at $E_{c 1}<E<E_{c 2}$.

It is convenient to normalize the electric field over $E_{2}$ and $j(E)$ over $j_{\text {ext }}$ introducing the dimensionless quantities $\mathcal{E} \equiv E / E_{2}$ and $u(\mathcal{E}) \equiv j(E) / j_{\text {ext }}$. Then, under certain assumptions, in the traveling coordinate frame connected with the domain, the normalized electric field in the sample is described by the following equation [19]:

$$
\mathcal{D} \mathcal{E}_{\xi \xi}+\alpha[s-u(\mathcal{E})] \mathcal{E}_{\xi}+[1-u(\mathcal{E})]=\mathcal{E}_{\eta}(\xi, \eta),
$$

where the subscripts indicate the corresponding derivatives. Eq. (1) is written in dimensionless variables, whose detailed definition is not important for the subsequent analysis (it may be found in Ref. [19]). Note only that $\mathcal{D}, s, \xi$, and $\eta$ stand for the diffusion coefficient, the domain velocity in the laboratory coordinate frame, traveling coordinate, and time, respectively; $\alpha=$ const $>0$ is the ratio of two characteristic spatial scales of the problem at $E=E_{2}$.

We suppose that $\mathcal{D}=$ const. This assumption simplifies calculations, but it is not crucial for the analysis. A more general case, when $\mathcal{D}=\mathcal{D}(\mathcal{E})$, was inspected by Knight and Peterson [18].

It is important to stress that, if the dependence $u(\mathcal{E})$ is not related to the specific shape of $j(E)$ shown in Fig. 2, Eq. (1) is nothing but a nonlinear diffusion equation of quite a general type describing a wide diversity of problems. Accordingly, the results discussed below may be applied to a much broader class of problems, provided these problems have traveling solitary-wave-type solutions.

For a steady-state traveling wave, the r.h.s of Eq. (1) vanishes, and the equation transforms into ODE. For the problem in question, a simple analysis reveals that its phase plane $\left(\mathcal{E}, \mathcal{E}_{\xi}\right)$ has three singular points situated at the $\mathcal{E}$ axis at $\mathcal{E}=\mathcal{E}_{1,2,3}$ corresponding to $E=E_{1,2,3}$ in Fig. 2. I remind that by definition $\mathcal{E}_{2} \equiv 1$ since $\mathcal{E}=E / E_{2}$. In the phase plane, a single traveling domain is described by a homoclinic path beginning in the saddle $\left(\mathcal{E}_{1}, 0\right)$, making a loop around the unstable focus $\left(\mathcal{E}_{2}, 0\right)$ and ending up in the same saddle $\left(\mathcal{E}_{1}, 0\right)$, see Fig. 3 .

It is possible to show that such a solution of Eq. (1) exists at $s=1$ solely [18]. Since this is the only case I am interested in, $s$ below is always supposed to be equal to unity. Then, the homoclinic path may be found explicitly [18]; however, I do not need this expression for the subsequent inspection. Let us just designate the steady-state solution of Eq. $(1)$ as $\mathcal{E}_{0}(\xi)$. The goal of this paper is to analyze the stability of this solution against small time-dependent perturbations $\delta \mathcal{E}(\xi, \eta)$. 


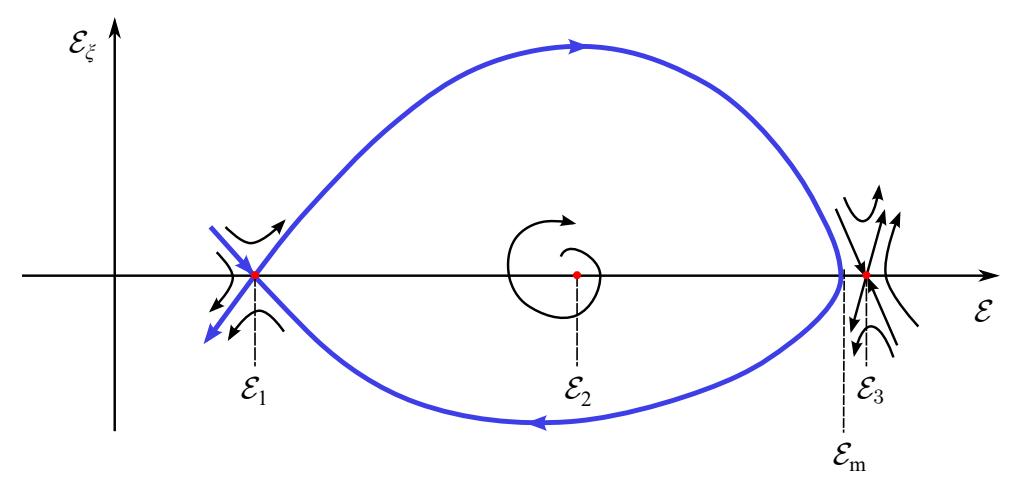

Figure 3. Phase plane $\left(\mathcal{E}, \mathcal{E}_{\tilde{\xi}}\right)$. Schematically. Three singular points are marked with red. The blue curve designates the homoclinic path corresponding to a single traveling domain. If the point $\left(\mathcal{E}_{\mathrm{m}}, 0\right)$ merges with $\left(\mathcal{E}_{3}, 0\right)$, the homoclinic path is split into two independent heteroclinic ones (the upper and lower parts of the homoclinic path, respectively). See the text for details.

\section{Stability Analysis}

The stability analysis performed in Ref. [18] generalizes a very beautiful approach developed by Zeldovich and Barenblatt for the inspection of the stability of a slow combustion front [24]. Its main idea is as follows. It is supposed that $\delta \mathcal{E}(\xi, \eta)=$ $\mathcal{E}^{(1)}(\xi) \exp (-\lambda \eta)$, where $\lambda$ is an eigenvalue of the stability problem. If there is a negative $\lambda$ in the problem's spectrum, it means instability.

Substituting $\mathcal{E}(\xi, \eta)=\mathcal{E}^{(0)}(\xi)+\mathcal{E}^{(1)}(\xi) \exp (-\lambda \eta)$ in Eq. (1) and linearizing the result in small $\mathcal{E}^{(1)}$, one arrives at the eigenvalue problem

$$
D \mathcal{E}_{\tilde{\xi}(1)}^{(1)}+\alpha\left[1-u\left(\mathcal{E}^{(0)}(\xi)\right)\right] \mathcal{E}_{\mathcal{\xi}}^{(1)}-u_{\mathcal{E}}\left(\mathcal{E}^{(0)}(\xi)\right) \mathcal{E}^{(1)}=-\lambda \mathcal{E}^{(1)},
$$

supplemented with the boundary conditions $\mathcal{E}^{(1)} \rightarrow 0$ at $\xi \rightarrow \pm \infty$. Then, introducing a new function $\psi(\xi)$ connected with $\mathcal{E}^{(1)}(\xi)$ by the relation

$$
\psi(\xi)=\exp \left(\frac{\alpha}{2 \mathcal{D}} \int\left(1-u\left(\mathcal{E}^{(0)}(\xi)\right) d \xi\right) \mathcal{E}^{(1)}(\xi) \equiv F(\xi) \mathcal{E}^{(1)}(\xi)\right.
$$

one reduces Eq. (2) to the standard Schrödinger equation ${ }^{4}$

$$
\begin{aligned}
& \hat{H} \psi=\Lambda \psi \\
& \hat{H}=-\frac{d^{2}}{d \xi^{2}}+V(\xi) \\
& V(\xi)=\left(\frac{\alpha\left(1-u\left(\mathcal{E}^{(0)}(\xi)\right)\right.}{2 \mathcal{D}}\right)^{2}+\left(1-\frac{\alpha \mathcal{E}_{\xi}^{(0)}(\xi)}{2}\right) \frac{u_{\mathcal{E}}\left(\mathcal{E}^{(0)}(\xi)\right)}{\mathcal{D}},
\end{aligned}
$$

where $\Lambda \equiv \lambda / \mathcal{D}$.

Note that since the homoclinic path begins and ends up at the same singular point $\left(\mathcal{E}_{1}, 0\right)$ and $u\left(\mathcal{E}_{1}\right)=1$, the considered steady-state traveling domain solution satisfies the condition $u\left(\mathcal{E}^{(0)}(\xi)\right) \rightarrow 1$ at $\xi \rightarrow \pm \infty$. Therefore, as it follows from Eq. (3), $\psi(\xi)$ and $\mathcal{E}^{(0)}(\xi)$ both have the same asymptotic behavior at $\xi \rightarrow \pm \infty$. This is important since it means, that none of the solutions of the Schrödinger equation generates "false" solutions of the initial stability problem, which may not satisfy the boundary conditions $\mathcal{E}^{(0)}(\xi) \rightarrow 0$ at $\xi \rightarrow \pm \infty$.

\footnotetext{
4 There is a misprint in the expression for $V(\xi)$ in Ref. [19] corrected in Eq. (6).
} 
Now the most essential part of the stability analysis begins. If $\mathcal{E}^{(0)}(\xi)$ is a solution of the steady-state version of Eq. (2), then, owing to the translational invariance of the problem $\mathcal{E}^{(0)}\left(\xi+\xi_{0}\right)$, where $\xi_{0}$ is any constant, also is a solution, i.e., being substituted in the 1.h.s. of Eq. (2), $\mathcal{E}^{(0)}\left(\xi+\xi_{0}\right)$, turns it to zero identically.

Let us consider the limit $\xi_{0} \rightarrow 0$. In this case, $\mathcal{E}^{(0)}\left(\xi+\xi_{0}\right) \approx \mathcal{E}^{(0)}(\xi)+\mathcal{E}_{\xi}^{(0)}(\xi) \xi_{0}$, and $\mathcal{E}_{\xi}^{(0)}(\xi) \xi_{0}$ here may be regarded as an infinitesimal perturbation to $\mathcal{E}^{(0)}(\xi)$. The perturbation transforms the steady-state solution into another steady-state solution. It means, such a perturbation is neutrally-stable and should not evolve in time. In other words, it means that $\mathcal{E}_{\xi}^{(0)}(\xi)$ is an eigenfunction of the stability problem with zero eigenvalue.

Note that we obtain this result based on the translational invariance solely, without the employment of a specific form of the differential operator in Eq. (2). These neutrallystable modes generated by a transformation of a continuous group of symmetry are called Goldstone modes. Since 2008, when the implementation of such a mode in stronginteraction physics ${ }^{5}$ brought about the Nobel Prize to Prof. Yoichiro Nambu, they are also called Nambu-Goldstone modes.

It is interesting to note that twenty-five years after the publication of Ref. [1] I returned to the inspection of the role of Goldstone modes in stability problems. This study ended up with the discovery of a new type of chaos at onset analogous to the second-order phase transitions in statistical physics, where the mean amplitudes of the turbulent modes played the role of the order parameter [25-27].

However, I have departed from the stability analysis of the Gunn domain. It is high time to be back. Actually, not so much remains to do. Collecting together all mentioned above, one can conclude that

$$
\psi(\xi)=F(\xi) \mathcal{E}_{\xi}^{(0)}(\xi)
$$

is the eigenfunction of Schrödinger equation, Eqs. (4)-(6) with zero eigenvalue.

Recall now the oscillation theorem [20]. It states that in a 1D Schrödinger equation $n$th wave function of a discrete spectrum should vanish $n$ times. Then, it is not so difficult to show that the integral in the exponent in Eq. (3) always remains finite, i.e., $F(\xi)$ never vanishes. Thus, all zeros of $\psi(\xi)$, if any, coincides with those of $\mathcal{E}_{\mathcal{\xi}}^{(0)}(\xi)$. Finally, since for the traveling domain the profile $\mathcal{E}^{(0)}(\xi)$ has a single maximum (the homoclinic path in the phase plane $\left(\mathcal{E}, \mathcal{E}_{\xi}\right)$ crosses the $\mathcal{E}$-axis at $\mathcal{E}=\mathcal{E}_{\mathrm{m}}$, situated in between $\mathcal{E}_{2}$ and $\left.\mathcal{E}_{3}\right)$, see Fig. 3), the product $F(\xi) \mathcal{E}_{\xi}^{(0)}(\xi)$ has a single zero at the value of $\xi$ corresponding to the maximal field achieved in the domain $\mathcal{E}_{\mathrm{m}}$. It means that the wave function Eq. (3) is the one for the first excited state. The "energy" of the ground state should be lower than those for excited states. Since the first excited state has zero "energy" this gives rise to the conclusion that the ground state has negative "energy", i.e., the spectrum of Eqs. (4)-(6) has a single negative eigenvalue. In other words, the solution $\mathcal{E}^{(0)}(\xi)$ is unstable with the instability increment equal to the modulus of $\lambda$, corresponding to the ground state of the Schrödinger equation.

Though the problem in question has several parameters, the actual control parameter, which relatively easily may be varied in an experiment, is the current in the circuit, $j_{\text {ext }}$. Varying $j_{\text {ext }}$ one can change the values of $E_{1,2,3}$ and hence the shape of the traveling domain.

At a certain value of $j_{\text {ext }}=j_{0}$ the maximal field in the domain, $\mathcal{E}_{\mathrm{m}}$ merges with $\mathcal{E}_{3}$, and the homoclinic path in the plane $\left(\mathcal{E}, \mathcal{E}_{\xi}\right)$ is split into two independent heteroclinic paths connecting the singular points $\left(\mathcal{E}_{1}, 0\right)$ and $\left(\mathcal{E}_{3}, 0\right)$. One of these paths lies entirely in the upper semi-plane. For this solution $\mathcal{E}_{\xi}^{(0)}$ is always positive at any finite $\xi$. The other lies entirely in the lower semi-plane and for it $\mathcal{E}_{\xi}^{(0)}<0$ at any finite $\xi$, see Fig. 3. Each of

\footnotetext{
Do you remember my remark about interconnections of different fields in physics?
} 
these solutions corresponds to a traveling charge layer transferring the sample from one steady-state to another steady-state.

It is important that since for the layers $\mathcal{E}_{\mathcal{\xi}}^{(0)}$ does not vanish at any finite $\xi$, the corresponding wave function given by Eq. (7) is the one of the ground state of the Schrödinger equation. It means that in contrast to the traveling domain the traveling layers are stable $[18,19]$.

\section{Spectrum of Schrödinger Equation}

Thus, to get the value of the instability increment and hence, the characteristic time for the traveling domain decomposition, one has to obtain the energy level for the ground state in the Schrödinger equation with the complicated potential given by Eq. (6). As it has been mentioned above, the main idea employed in Ref. [1] to fulfill this task is to approximate the actual smooth potential by a superposition of square potential wells. The parameters of the wells are selected so that the approximated potential keeps all main features of the initial smooth profile. One of the approximation parameters remains free. It is fixed by the condition that the for the first excited state $\Lambda=0$.

This procedure reduces the solution of the complicated initial problem to finding the roots of a set of transcendental equations. In the worst case, the latter may be readily done numerically. To illustrate this rather a general approach, a simple case of a broad traveling domain is discussed below.

The domain becomes broad when $j_{\text {ext }}$ approaches at $j_{0}$. In the phase plane, it corresponds to the shift of the regular point of the path $\left(\mathcal{E}_{\mathrm{m}}, 0\right)$ toward the saddle $\left(\mathcal{E}_{3}, 0\right)$. In this case, the domain approximately may be presented as a nonlinear superposition of two layers with opposite charges, and the potential (6) has a shape of two wells separated by a barrier.

Each well is associated with the corresponding layer. The width of the barrier corresponds to the distance between the layers and, in the case under consideration, is large. Then, the tunneling through the barrier is exponentially weak. If the tunneling were suppressed entirely, each well would have corresponded to the potential generated by a single layer and, in accord with the said above, had the ground state with $\Lambda=0$. Thus, it is clear that the level with $\Lambda<0$ for the domain occurs owing to the splitting due to finite tunneling of the ground states in the two wells with the same value of $\Lambda=0$. Therefore, instead of the employment of rather a cumbersome general procedure of the solution of an entire problem with the finite tunneling, I, firstly, neglect the tunneling and consider the solutions of the Schrödinger equation in each square well separately. Then, finite tunneling is taken into account with the help of perturbation theory.

The values of the parameters of the square wells approximating the smooth profile of the potential may be obtained by inspection of Eq. (6). For example, bearing in mind that at $\xi \rightarrow \pm \infty$ the solution $\mathcal{E}^{(0)}(\xi)$ describing the domain satisfies the conditions $\mathcal{E}^{(0)} \rightarrow \mathcal{E}_{1} ; \mathcal{E}_{\tilde{\xi}}^{(0)} \rightarrow 0$ and that by definition $u\left(\mathcal{E}_{1,2,3}\right)=1$, one immediately obtains that both outer walls of the wells have the height equals $u_{\mathcal{E}}\left(\mathcal{E}_{1}\right) / \mathcal{D}$. Analogously, the barrier height is $u_{\mathcal{E}}\left(\mathcal{E}_{\mathrm{m}}\right) / \mathcal{D} \approx u_{\mathcal{E}}\left(\mathcal{E}_{3}\right) / \mathcal{D}$. The widths of the wells and the barrier are estimated based on the mentioned exact solution describing the domain path in the phase plane. The last remaining parameters, namely the depths of the wells are fixed by the conditions that the ground state in each well has $\Lambda=0$.

I do not present here these simple but cumbersome calculations. Just note that the problem is rather robust against errors in the approximation of $V(\xi)$. The robustness is related to the smallness of the split of the ground levels in the wells due to the tunneling and the fact that at the employed approach the important condition $\Lambda=0$ for the ground states in each well separately holds automatically.

Thus, I suppose that the wave functions of the ground state for each well $|1,2\rangle$ are known and that these wave functions satisfy the equations $\hat{H}_{1,2}|1,2\rangle=0$. Here $\hat{H}_{1,2}$ designate the Hamiltonians, whose potentials $V_{1,2}$ include the corresponding single well 


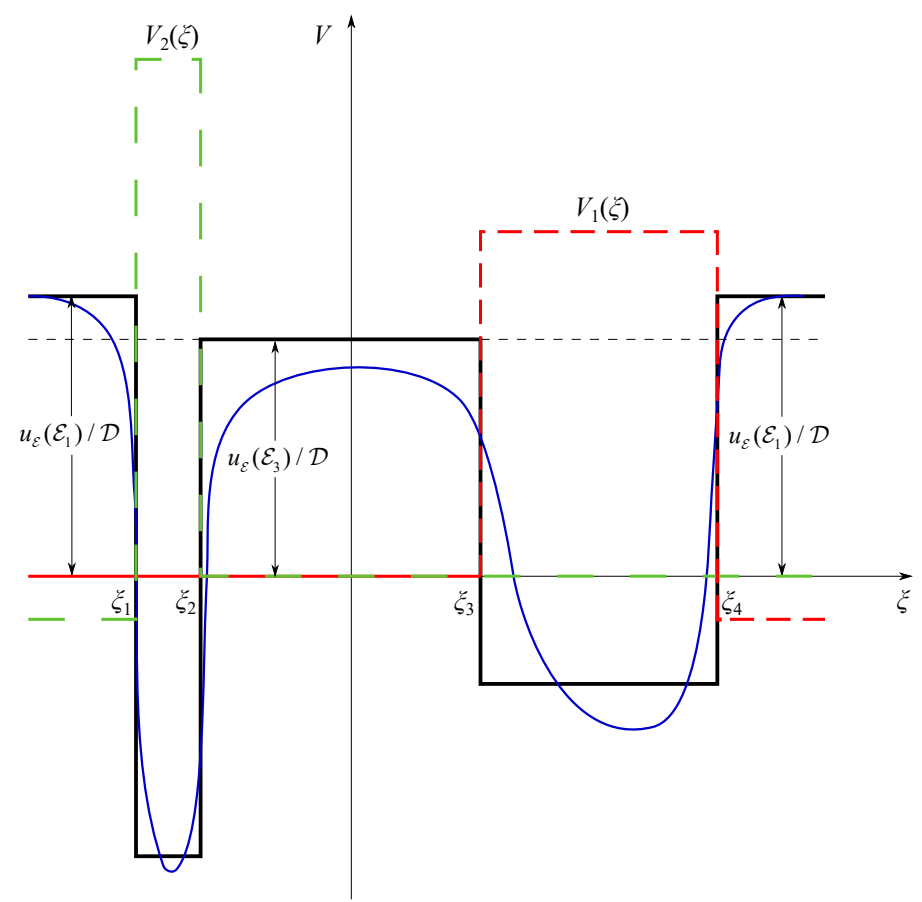

Figure 4. Schematically: The actual double-well potential $V(\xi)$ (smooth blue line). Its approximation by the double-well square potential $V_{D W S}(\xi)$ is shown in black; $\xi_{1,2,3,4}$ designate the coordinates of the walls of the wells. $U_{1,2}(\xi)=V_{D W S}(\xi)+V_{1,2}(\xi)$ are the potentials of the singlewell approximation, when tunneling is neglected.

solely. Then, I look for the wave function of the complete problem with the two-well potential in a form of a linear superposition of $|1,2\rangle$ :

$$
|\psi\rangle=c_{1}|1\rangle+c_{2}|2\rangle,
$$

where $c_{1,2}$ are constants, which should be defined in the course of calculations. Next, since $|\psi\rangle$ is an eigenfunction of the complete Hamiltonian $\hat{H}$

$$
c_{1} \hat{H}|1\rangle+c_{2} \hat{H}|2\rangle=\Lambda\left(c_{1}|1\rangle+c_{2}|2\rangle\right) .
$$

Making scalar products with $\langle 1,2|$ and taking into account the normalization conditions $\langle 1 \mid 1\rangle=\langle 2 \mid 2\rangle=1$, one arrives from Eq. (9) to the following equations for $c_{1,2}$ :

$$
\begin{aligned}
& \left(H_{11}-\Lambda\right) c_{1}+\left(H_{12}-\Lambda\langle 1 \mid 2\rangle\right) c_{2}=0 \\
& \left(H_{21}-\Lambda\langle 2 \mid 1\rangle\right) c_{1}+\left(H_{22}-\Lambda\right) c_{2}=0
\end{aligned}
$$

where $H_{11}, H_{12}, H_{21}$ and $H_{22}$ stand for the corresponding matrix elements. Note that the wave functions $|1\rangle$ and $|2\rangle$ are not orthogonal since they are the eigenfunctions of the different Hamiltonians, namely $\hat{H}_{1}$ and $\hat{H}_{2} \neq \hat{H}_{1}$.

The solvability condition requires vanishing of the determinant of Eqs. (10)-(11). This results in a quadratic equation for $\Lambda$. The difference between its two roots $\Delta=$ $\left|\Lambda_{1}-\Lambda_{2}\right|$ gives the desired instability increment.

This result has completed the instability analysis. However, the exact expression for $\Delta$ is rather cumbersome. Therefore, it is worth to simplify this result, employing smallness of certain parameters in Eqs. (10)-(11). To this end, I have to estimate the matrix elements and the overlap integrals $\langle 1,2 \mid 2,1\rangle$.

To calculate the matrix elements, it is convenient to single out from the full doublewell square potential $V_{D W S}(\xi)$ the part corresponding to a single well, i.e. to suppose 
that $V_{D W S}(\xi)=U_{1,2}-V_{1,2}$, see Fig. 4, where for the first well (left in Fig. 4) $V_{1}=0$ at $\xi<\xi_{3}$, while at $\xi>\xi_{3}$ the sum of $V_{D W S}$ and $V_{1}$ equals the height of the barrier. Analogously, for the second well (right) the sum $V_{D W S}+V_{2}$ equals the height of the barrier at $\xi<\xi_{2}$, while at $\xi>\xi_{2}$ the potential $V_{2}=0$. Thus, $U_{1,2}$ are the single-well potentials, and the corresponding Hamiltonians acting on their wave functions produce zero. Then, the leading terms in the matrix elements are estimated as follows:

$$
H_{11}=-\left\langle 1\left|V_{1}\right| 1\right\rangle \sim H_{22}=-\left\langle 1\left|V_{2}\right| 1\right\rangle \sim \exp \left[-2 d_{b} \sqrt{u_{\mathcal{E}}\left(\mathcal{E}_{3}\right) / \mathcal{D}}\right],
$$

where $d_{b}=\xi_{3}-\xi_{2}$ is the barrier width. The same estimate is true for $H_{22}$ (for the sake of simplicity I suppose that $d_{1,2} \sqrt{u_{\mathcal{E}}\left(\mathcal{E}_{3}\right) / \mathcal{D}}$ are not small, where $d_{1,2}$ stand for the widths of the corresponding wells). In the same manner one obtains

$$
H_{12} \sim H_{21} \sim\langle 2 \mid 1\rangle \sim\langle 1 \mid 2\rangle \sim \exp \left[-d_{b} \sqrt{u_{\mathcal{E}}\left(\mathcal{E}_{3}\right) / \mathcal{D}}\right],
$$

Then, in the leading approximation

$$
\Delta \approx H_{12}+H_{2,1} \sim \exp \left[-d_{b} \sqrt{u_{\mathcal{E}}\left(\mathcal{E}_{3}\right) / \mathcal{D}}\right] .
$$

Thus, as it could be expected, the value of the instability increment for the broad domain is exponentially small indeed.

Finally, note that for each single square well the normalized wave functions $|1,2\rangle$ may be readily obtained in the explicit form, including the normalization constant. Thus, it is just a matter of more or less routine calculations to improve the accuracy of Eq. (14), taking into account the prefactors and dropped higher-order exponentially small terms.

\section{Conclusions}

Summarizing and generalizing the discussed above, one may arrive at the following conclusions:

- The analysis of the linear stability of traveling wave solutions in a wide class of nonlinear diffusion probelms is reduced to the inspection of a bottom part of the spectrum of the associated Schrödinger equation, whose potential is generated by the profile of the analyzed solution.

- The translational invariance transformation generates in the stability spectrum a neutrally-stable (Goldstone) mode.

- The qualitative answer to the question about the stability of the solution is readily obtained based on the oscillation theorem - if the Goldstone mode does not have any nodes, the solution is stable. Otherwise, it is unstable.

- To quantitatively characterize the instability (if any), the "energy" level of the ground state of the Schrödinger equation should be obtained.

- A powerful tool to make the problem of a bottom part of the Schródinger equation spectrum tractable is to approximate the potential by square wells.

These conclusions are rather general. They are valid far beyond the frameworks of the Gunn effect and hopefully may help to analyze the stability of traveling waves in a broad class of nonlinear diffusion problems.

The developed approach to find an approximate solution and spectrum of the Schrödinger equation with a complicated potential valid for ground and low-excited states may be regarded as a complement to the well-known WKB method, which is good in the opposite case of high-excited states. A common disadvantage of both approaches is the approximation error, which is difficult to improve and even control.

\section{Acknowledgement}

I am very grateful to the entire Editorial staff of Physics for this Special Issue dedicated to my humble person. My special thanks to my friends and colleagues Boris 
Malomed, Andrey Miroshnichenko, and Fernando Moreno, who volunteered to be Guest Editors of the Special Issue and excellently completed this difficult task. Thank you very much indeed!

This work is financed by the Moscow Engineering Physics Institute Academic Excellence Project (agreement with the Ministry of Education and Science of the Russian Federation of 27 August 2013, Project No. 02.a03.21.0005).

\section{References}

1. Tribel'skii, M. Gunn domain instability at fixed current (in Russian). Fizika i Tekhnika Poluprovodnikov 1971, 5, 2033.

2. El'yashevich, M.A. In memory of Mikhail Aleksandrovich El'yashevich. Physics-Uspekhi 1996, $39,853$.

3. Dzyaloshinskii, I.E.; Zakharov, V.E.; Inogamov, N.A.; Kolokolov, I.V.; Kuznetsov, E.A.; Lebedev, V.V.; Novikov, S.P.; Sagdeev, R.Z.; Starobinskii, A.A.; Stishov, S.M.; Fortov, V.E.; Khalatnikov, I.M. In memory of Sergei Ivanovich Anisimov. Physics-Uspekhi 2019, $62,1282-1283$.

4. Khalatnikov, I.M. History of the Landau Institute. https://www.itp.ac.ru/en/about/history/.

5. Grosberg, A.Y.; Kaganov, M.I.; Tribel'skii, M.I.; Khokhlov, A.R. Il'ya Mikhailovich Lifshits (on the 80th anniversary of his birth). Phys. Usp. 1997, 40, 225-226. doi:10.1070/PU1997v040n02ABEH000208.

6. P.L. Kapitza Institute for Physical Problems of Russian Academy of Sciences. https://www.kapitza.ras.ru/index.php?cont=hist\&lang=en.

7. Mark Azbel. https://en.wikipedia.org/wiki/Mark_Azbel.

8. Aleksandrov, A.P.; Velikhov, E.P.; Ginzburg, V.L.; Zel'dovich, Y.B.; Kadomtsev, B.B.; Lifshitz, E.M.; Prokhorov, A.M.; Rytov, S.M.; Sagdeev, R.Z.; Shafranov, V.D. Mikhail Aleksandrovich Leontovich (Obituary). Phys. Usp. 1981, $24,730-731$. doi:10.1070/PU1981v024n08ABEH004857.

9. Gunn, J.B. Microwave oscillations of current in III-V semiconductors. Solid State Communications 1963, 1, 88-91.

10. Solymar, L.; Walsh, D.; Syms, R. Principles of semiconductor devices; Oxford University Press.

11. Hsu, H.W.; Dominguez, M.J.; Sih, V. Gunn threshold voltage characterization in GaAs devices with wedge-shaped tapering. Journal of Applied Physics 2020, 128, 074502.

12. Hajo, A.S.; Yilmazoglu, O.; Dadgar, A.; Küppers, F.; Kusserow, T. Reliable GaN-Based THz Gunn Diodes with side-contact and field-plate technologies. IEEE access 2020, 8, 84116-84122.

13. Semyonov, E.V.; Malakhovskij, O.Y. Short-Pulse Properties of the Gunn Diode. IEEE Transactions on Electron Devices 2020, $67,2100-2105$.

14. Novoa-López, J.; Paz-Martínez, G.; Sánchez-Martín, H.; Lechaux, Y.; Íñiguez-de-la Torre, I.; González, T.; Mateos, J. Temperature behavior of Gunn oscillations in planar InGaAs diodes. IEEE Electron Device Letters 2021.

15. Liang, F.; Wang, H.; Pan, J.; Li, J.; Xu, K.; Luo, Y. Voltage-driven mutual phase locking of planar nano-oscillators. International Journal of Modern Physics B 2021, 35, 2150072.

16. Bonilla, L.L.; Teitsworth, S.W. Nonlinear wave methods for charge transport, Chapter 6; John Wiley \& Sons, 2009.

17. Kroemer, H. Theory of the Gunn effect. Proceedings of the IEEE 1964, 52, 1736-1736.

18. Knight, B.; Peterson, G. Theory of the Gunn effect. Physical Review 1967, 155, 393.

19. Volkov, A.F.; Kogan, S.M. Physical phenomena in semiconductors with negative differential conductivity. Phys. Usp. 1969, 11, 881-903. doi:10.1070/PU1969v011n06ABEH003780.

20. Landau, L.D.; Lifshitz, E.M. Quantum mechanics: non-relativistic theory, §20, §21; Vol. 3, Elsevier, 2013.

21. Lifshits, I.M.; Rzhevskii, V.V.; Tribel'skii, M.I. Nonlinear Acoustic Effects in Metals Near the Electron Topological Transition Point. Zh. Eksp. Teor. Fiz. 1981, 81, 1528-1541.

22. Andreev, A.F.; Brazovskii, S.A.; Volovik, G.E.; Kats, E.I.; Kondratenko, P.S.; Kreines, N.M.; Mineev, V.P.; Novikov, S.P.; Obukhov, S.P.; Pitaevskii, L.P.; Khmel'nitskii, D.E.; Eliashberg, G.M. Igor Ekhiel'evich Dzyaloshinskii (on his 90th birthday). Physics-Uspekhi 2021, 64, 214-215.

23. Tribel'skii, M.I. Gunn domain instability increment. Sov. Phys. Solid Sate 1974, 15, 2448.

24. Zeldovich, Y.B.; Barenblatt, G.I. Theory of flame propagation. Combustion and flame 1959, 3, 61-74.

25. Tribelsky, M.I.; Tsuboi, K. New scenario for transition to turbulence? Physical review letters 1996, 76, 1631.

26. Hidaka, Y.; Huh, J.H.; Hayashi, K.i.; Kai, S.; Tribelsky, M.I. Soft-mode turbulence in electrohydrodynamic convection of a homeotropically aligned nematic layer. Physical Review E 1997, 56, R6256.

27. Tribel'skii, M.I. Short-wavelength instability and transition to chaos in distributed systems with additional symmetry. PhysicsUspekhi 1997, 40, 159-180. 